Universidade Tecnológica Federal do Paraná - UTFPR

Campus Ponta Grossa - Paraná - Brasil

ISSN: 1981-3686/v. 05, n. 1 suplemento: p. 379-386, 2011

D.O.I.:10.3895/S1981-36862011000100001S1
Revista Brasileira de Tecnologia

Agroindustrial

\title{
DESENVOLVIMENTO DE FERMENTADORES EM SÉRIE PARA O ESTUDO CINÉTICO DE FERMENTADO DE MAÇÃ
}

\section{DEVELOPMENT OF FERMENTATOR IN SERIES FOR THE STUDY OF KINETIC APPLE FERMENTED}

\author{
Frederico Koch Fernandes de Souza ${ }^{1}$, Renan Oliveira da Luz ${ }^{1}$, Caroline Mongruel Eleutério dos Santos ${ }^{1}$, \\ Giovana de Arruda Moura Pietrowski ${ }^{2}$, Gilvan Wosiacki ${ }^{1}$, Alessandro Nogueira ${ }^{1}$ \\ ${ }^{1}$ Universidade Estadual de Ponta Grossa - UEPG - Ponta Grossa - Brasil \\ fredericovonkoch@yahoo.com.br \\ ${ }^{2}$ Universidade Tecnológica Federal do Paraná - UTFPR - Ponta Grossa - Brasil \\ gampietrowski@brturbo.com.br
}

\begin{abstract}
Resumo
Em busca do melhoramento do fermentado de maçã brasileiro, este trabalho objetivou a implementação de um sistema de fermentadores em série com possibilidade de estabelecer as condições do processo, monitoramento do processo de fermentação alcoólica e avaliação de diferentes cepas comerciais de leveduras Saccharomyces cerevisiae na obtenção de fermentado de maçã. As cepas de leveduras utilizadas foram a Arome Plus, Bouquet e Fermol Aromatic, do fabricante (AEB Group) e a Saccharomyces cerevisiae UFLA CA - 11. Os fermentadores foram confeccionados em vidro com volume de 1,45 L e volume útil de 1,20 L, com dimensões e materiais que possibilitassem a esterilização em autoclave. Os fermentadores foram montados em sala com temperatura controlada $\left(20^{\circ} \mathrm{C}\right)$ e interligados a uma tubulação de silicone para saturação com dióxido de carbono a fim de manter a anaerobiose, com controle de pressão e fluxo do gás carbônico, de modo a permitir coletas através da elevação da pressão no sistema, uma excelente ferramenta para realização do projeto. O sistema (4 fermentadores) possibilitou avaliar em quadruplicata cada levedura nas mesmas condições de fermentação. A coleta máxima de amostra corresponde a $20 \%$ do volume útil. Este volume foi suficiente para análises físico-químicas e microbiológicas ao longo do período de fermentação (10 dias) até exaustão dos açúcares fermentescíveis. $O$ sistema de fermentadores em série foi uma excelente ferramenta para avaliação de parâmetros fermentativos na elaboração de bebidas fermentadas.
\end{abstract}

Palavras chave: Saccharomyces sp., maçã, fermentado, fermentador.

\section{Introdução}

O Brasil tem registrado um aumento significativo na produção de maçãs. Nas últimas décadas, a produção cresceu cerca de $6.000 \%$. Na safra de 1977/78 foram produzidas em torno de 
14 mil toneladas e em 2007/08 esse número ultrapassou 900 mil toneladas (EPAGRI, 2002; WOSIACKI et al., 2008). As frutas são selecionadas e encaminhas ao consumidor para atender a demanda in natura. Segundo Paganini et al. (2004) até 30\% dessa produção se torna material de descarte, devido a injúrias causadas por fatores climáticos e ou fitopatológicos, que então é processado pelo setor agro industrial a fim de agregar valor em produtos nobres como sucos (clarificado, néctares e concentrado) ou produtos fermentados (sidra e vinagre) e destilados (eaude-vie e calvados).

Com relação ao fermentado de maçã, nota-se que houve uma diminuição no consumo, devido à baixa qualidade do produto encontrado no Brasil. A elaboração da sidra brasileira contém alta concentração de sulfito, que acaba afetando a formação de aromas por leveduras oxidativas ou não-convencionais presentes na epiderme das frutas. Somado a isso, uma fermentação acelerada e adição de elevada concentração de açúcar, afetam ainda mais a qualidade do produto (NOGUEIRA e WOSIACKI, 2010).

Dessa forma, para restaurar e ampliar a fatia do mercado correspondente ao fermentado de maçã, é necessário a melhoria de suas qualidades organolépticas. A cepa de levedura utilizada é um fator determinante em diversas características do produto. Vários estudos relacionados ao melhoramento genético de cepas Saccharomyces cerevisae, tiveram como objetivo, uma rápida fermentação, elevado rendimento alcoólico e tolerância ao dióxido de enxofre na elaboração de vinhos e cervejas (PRETORIUS, 2000; VALERO et al., 2005).

Atualmente, tem-se utilizado cepas provindas da enologia, adequadas à fabricação de vinhos a base de uvas. Não existem trabalhos relacionados à caracterização de cepas especificas para indústria sidrícola nacional, o que acaba comprometendo a qualidade do fermentado de maçã.

Sendo assim, este trabalho tem como objetivo implantar um sistema de fermentação em série e avaliar diferentes cepas comerciais de leveduras Saccharomyces na elaboração de fermentados de maçãs, bem como caracterizar a aptidão destas cepas na formação de bioaromas que sejam atrativos sensorialmente ao consumidor.

\section{Material e Métodos}

Foram utilizados $(90 \mathrm{~kg}$ ) de maçãs Gala obtidas no comércio local de Ponta Grossa, Paraná. As leveduras testadas foram as Saccharomyces cerevisiae: Arome Plus, Bouquet e Fermol Aromatic, do fabricante (AEB Group) e a Saccharomyces cerevisiae UFLA CA - 11 do fabricante LNF. Os reativos e padrões utilizados foram de qualidade pro analysis.

O processamento da maçã para obtenção do mosto foi realizado, de acordo com procedimentos laboratoriais tais como: sanitização com hipoclorito de sódio $\left(150 \mathrm{mg} \mathrm{L}^{-1}\right.$ por 5 
min. a $5{ }^{\circ} \mathrm{C}$ ), trituração (Multiprocessador de alimentos Metvisa, Tipo MPA, disco $\mathrm{n}^{\circ} 4$ ), prensagem 3,0 $\mathrm{kgf} \mathrm{cm}^{-2}$ durante 3 minutos (Prensa hidráulica Eureka, Hoppe Ind. Ltda.), despectinização enzimática com pectinase (Pectinex Ultra SP, Novozymes do Brasil) 3,0 mL hL'1 (120 min. $20-25^{\circ} \mathrm{C}$ ), após a reação foi feita a trasfega, filtragem em papel filtro, envase seguido de lacre em garrafas de $660 \mathrm{~mL}$ em vidro âmbar e pasteurizadas a $80^{\circ} \mathrm{C}$ durante 20 minutos, segundo (WOSIACKI et al., 1989).

Para que fosse possível a padronização das condições na realização dos experimentos, foi necessária a implantação de fermentadores em vidro com volume de 1,45 L e volume útil de 1,2 L, com dimensões e materiais que possibilitassem a esterilização em autoclave. Os fermentadores foram montados em sala com temperatura controlada $\left(20^{\circ} \mathrm{C}\right)$ e interligados a uma tubulação de silicone para saturação com dióxido de carbono a fim de manter a anaerobiose, com controle de pressão e fluxo do gás carbônico, de modo a permitir coletas através da elevação da pressão no sistema, conforme a Figura 1.

Figura 1 - Esquema dos fermentadores em série em condição de anaerobiose

(4)

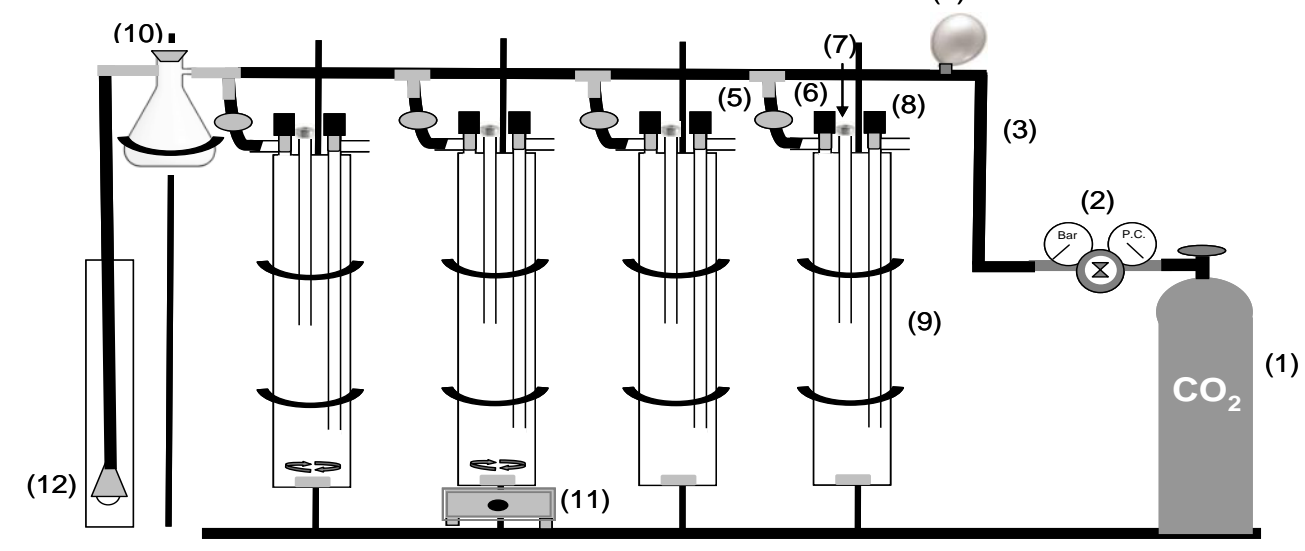

(1): Cilindro de gás carbônico para pressurizar sistema. (2): válvula de baixa pressão (Famabras). (3): Tubulação de passagem de gás carbônico pelo sistema. (4): Válvula de segurança. (5): Filtro estéril de $0,20 \mu \mathrm{m}$ (Millipore). (6): Tampa rosca para entrada e saída de gás no fermentador. (7): Tampa de borracha para inoculação das leveduras no mosto. (8): Tampa rosca para retirada de amostra. (9): Fermentador de $1450 \mathrm{~mL}$. (10): Recipiente de segurança contra retorno de água no sistema de fermentação. (11): Agitador magnético (Quinis). (12): Batoque para manutenção da anaerobiose no sistema de fermentação.

Para os ensaios de fermentação o mosto foi transferido para os 4 fermentadores (ensaios em quadruplicata). A levedura foi rehidratada, conforme recomendação do fabricante, e inoculada com uma população inicial de aproximadamente $2,0 \times 10^{6} \mathrm{ufc} \cdot \mathrm{mL}^{-1}$.

Para a coleta de amostra o fermentado era homogeneizado (uma barra magnética foi deixada em cada fermentador) pelo agitador magnético (Quinis). Foram estabelecidas algumas normas para assegurar a assepsia e homogeneidade das coletas, tais como: Limpeza da válvula de saída (8) com álcool 70\% antes e depois de cada coleta, descarte dos primeiros 7-10 mL provenientes da haste, tempo de agitação de no mínimo 20 minutos e frasco coletor estéril. 
Após terem sido devidamente homogeneizadas, as amostras foram retiradas com $0,1,2,3$, 5, 7 e 10 dias de fermentação. Para tanto, o fermentador é munido de uma haste, que mediante aumento da pressão interna de $\mathrm{CO}_{2}$, adicionado ou produzido pelas leveduras, dependendo do momento da fermentação alcoólica, conduz o fermentado até a saída (8). Como o fluxo é unidirecional e a haste é longa o suficiente, a coleta é feita sem qualquer tipo de contaminação. A amostra era utilizada para a contagem de leveduras e centrifugação para retirada das leveduras e congelamento da amostra para análises físico-químicas e instrumentais posteriores.

\section{Análises físico-químicas}

Nos pontos 0, 3, 5, 7 e 10 (dias) foram realizadas análises de açúcares redutores (AR) quantificados pelo método químico clássico de Somogyi-Nelson, assim como os açúcares redutores totais (ART) após a hidrólise da sacarose com $\mathrm{HCl} 1 \mathrm{~N}\left(50^{\circ} \mathrm{C} / 5\right.$ minutos) e álcool através do uso do ebuliômetro. A acidez titulável (AT) total foi determinada por neutralização com $\mathrm{NaOH} 0,1 \mathrm{~N}$ até pH 8,33 com fenolftaleína e calculada como ácido málico, sendo expresso em g $100 \mathrm{~mL}^{-1}$, e a acidez volátil (AV) foi calculada como ácido acético e expressa em g 100 $\mathrm{mL}^{-1}$ (IAL, 2008). O nitrogênio total foi determinado pelo método de Kjedhal (BARON et al., 1977; IAL, 2008).

\section{Análises microbiológicas}

As amostras dos fermentados de maçã foram coletadas e preparadas para contagem microbiológica conforme recomendado por Silva et al. (2010). Foram preparadas diluições seriadas em água peptonada a 0,1\%, e semeadas em superfície, com alça de Drigalski, no meio seletivo para leveduras YMA - Yeast Malt Agar. Estas análises foram realizadas no Laboratório de Microbiologia da Universidade Tecnológica Federal do Paraná - Campus de Ponta Grossa.

\section{Resultados e Discussão}

Na Tabela 1 pode ser observado que o teor de nitrogênio, que na maçã está na forma de aminoácidos, classifica o mosto da cultivar Gala com elevado teor (>150 mg L $\left.\mathrm{m}^{-1}\right)$ sendo suficiente para uma completa exaustão dos açúcares (DRILLEAU et al., 1991; ALBERTI et al., 2011). O mosto de maçã da cultivar Gala apresentou baixo teor de açúcares $\left(9,78 \mathrm{~g} 100 \mathrm{~mL}^{-1}\right)$ atestando que o grau de maturidade da fruta utilizada para o processamento não estava no seu estádio completo de maturação o que pode afetar o rendimento de etanol. O teor de ácido málico do mosto da cultivar 


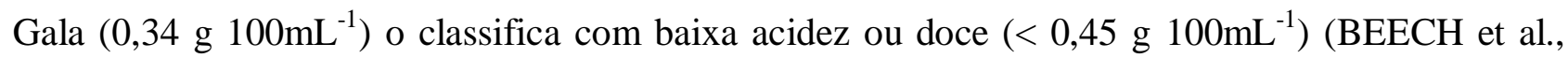
1972).

Na Tabela 1, após 10 dias de fermentação pode ser analisada a evolução dos compostos do mosto no fermentado com diferentes cepas de leveduras fermentativas. Pode-se verificar um aumento na acidez volátil para todas as cepas provavelmente devido à síntese de ácido acético e acetato de etila (CSOMA e SIPICZKI, 2008). O consumo de nitrogênio foi ao redor de $90 \mathrm{mg} \mathrm{L}^{-1}$, sendo próximo ao encontrado na literatura (NOGUEIRA et al., 2007).

Tabela 1 - Resultados das análises físico-químicas do mosto e dos diferentes fermentados de maçã da cultivar Gala

\begin{tabular}{lccccc}
\hline Parâmetros físico-químicos & Mosto & \multicolumn{4}{c}{ Leveduras comerciais } \\
\cline { 3 - 6 } & cv. Gala & Bouquet & Arôme Plus & F. Aromatic & UFLA CA-11 \\
\hline Nitrogênio $\left(\mathrm{mg} \mathrm{L}^{-1}\right)$ & $153,47 \pm 0,02$ & $65,64 \pm 6,54$ & $63,79 \pm 0,00$ & $64,57 \pm 5,34$ & $62,87 \pm 1,30$ \\
AT $\left(\mathrm{g} 100 \mathrm{~mL}^{-1}\right)$ & $0,34 \pm 0,004$ & $0,33 \pm 0,01$ & $0,35 \pm 0,01$ & $0,34 \pm 0,01$ & $0,26 \pm 0,01$ \\
AV $\left(\mathrm{g} 100 \mathrm{~mL}^{-1}\right)$ & $0,02 \pm 0,008$ & $0,05 \pm 0,01$ & $0,03 \pm 0,01$ & $0,04 \pm 0,01$ & $0,03 \pm 0,01$ \\
AR $\left(\mathrm{g} 100 \mathrm{~mL}^{-1}\right)$ & $9,23 \pm 0,09$ & $1,38 \pm 0,04$ & $0,99 \pm 0,05$ & $1,10 \pm 0,02$ & $0,79 \pm 0,05$ \\
ART $\left(\mathrm{g} 100 \mathrm{~mL}^{-1}\right)$ & $9,78 \pm 0,05$ & $1,58 \pm 0,01$ & $2,48 \pm 0,02$ & $1,31 \pm 0,01$ & $0,91 \pm 0,01$ \\
Etanol $\left({ }^{\circ} \mathrm{GL}\right)$ & - & 5,6 & 5,2 & 5,3 & 5,5
\end{tabular}

Nota: AT: acidez total; AV: acidez volátil; AR: açúcar redutor; ART: açúcar redutor total.

O teor de açúcares residuais foi baixo, considerando que todas as cepas apresentaram graduação alcoólica considerada boa para o produto. Estes açúcares melhoram a qualidade da bebida, adequando-se mais ao perfil de paladar do brasileiro que tem preferência por bebidas doces. Percebe-se que as cepas atingiram teores similares no produto final, apresentando apenas uma cinética diferente no decorrer do processo. Permaneceram açúcares residuais no produto, demonstrando uma tendência de atingir maiores valores de álcool caso o processo não tivesse sido interrompido.

A cepa $S$. cerevisiae Bouquet foi a que apresentou maior teor alcoólico $\left(5,6^{\circ} \mathrm{GL}\right)$ e a cepa $S$. cerevisiae UFLA CA-11 foi a que consumiu maior teor de açúcares permanecendo um residual de $0,91 \mathrm{~g} 100 \mathrm{~mL}^{-1}$. Este fato pode ser devido à produção de metabólitos secundários do processo fermentativo. A fermentação foi realizada em condições próximas as observadas no processamento de fermentado de maçã. A temperatura variou de $19-21^{\circ} \mathrm{C}$, oscilação e temperaturas comuns, uma vez que o fermentado de maçã é elaborado no verão. 
Figura 2 - Resultados de contagens de células dos fermentados sob ação de quatro diferentes leveduras fermentativas

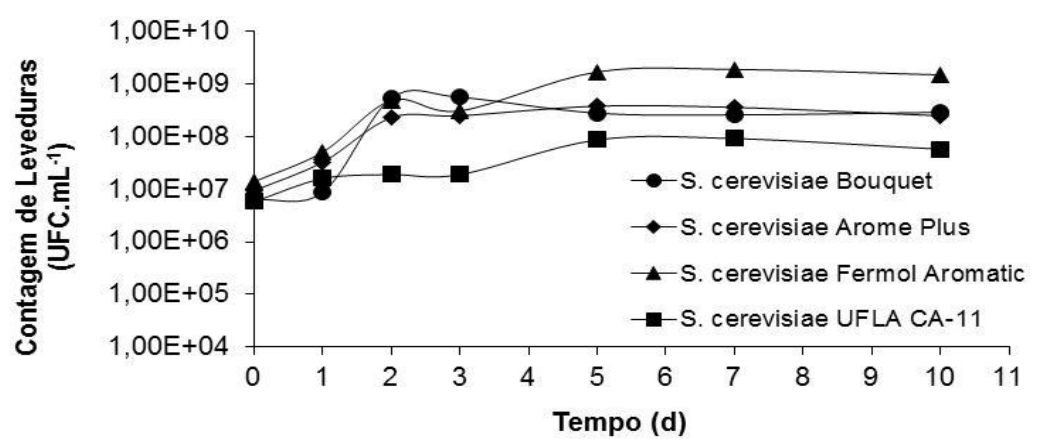

Na Figura 2, percebe-se que as populações máximas atingidas pelas leveduras são consideradas altas $\left(10^{8} \mathrm{ufc} \mathrm{mL}^{-1}\right)$ para produtos fermentados de maçã, pois diversos estudos revelam

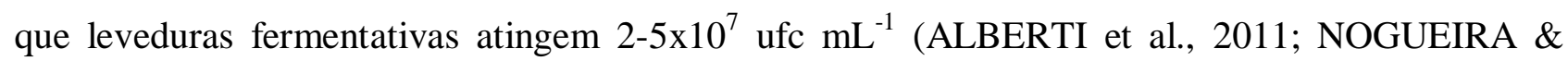
WOSIACKI, 2010). Acredita-se que a pasteurização realizada no mosto pode ter colaborado para tal fato, pois com a redução da microbiota natural, as cepas de Saccharomyces sp. puderam atingir populações maiores, por não ter encontrado as formas microbianas que normalmente as acompanham, sendo seus competidores naturais (NOGUEIRA et al., 2008; WIECHETECK et al., 2005; DIERINGS et al., 2008).

Todas as cepas atingiram velocidades máximas de fermentação ao redor do segundo dia de processo (Tabela 2). Sendo que a cepa que alcançou a maior $\mathrm{V}_{\max }$ foi UFLA CA-11 em 1,75 dias de fermentação e a cepa que demorou mais para alcançar este parâmetro foi a Fermol Aromatic (2,25 dias). Apesar de a cepa Bouquet ter atingido o maior número de células (aproximadamente $10^{9}$ ufc $\mathrm{mL}^{-1}$ ) esta não atingiu a maior velocidade máxima, ficando com $9,59 \mathrm{gCO}_{2} \mathrm{~L}^{-1} \mathrm{~d}^{-1}$.

Tabela 2 - Resultados de velocidade máxima dos fermentados de maçã produzido

\begin{tabular}{lll}
\hline Cepas & V máx $\left(\mathbf{g C O}_{2} \cdot \mathbf{L}^{-1} \cdot \mathbf{d}^{-1}\right)$ & Tempo v máx $($ dias $)$ \\
\hline Bouquet & 9,59 & 2,00 \\
Arome Plus & 8,27 & 1,75 \\
UFLA CA-11 & 9,83 & 1,75 \\
Fermol Aromatic & 9,64 & 2,25 \\
\hline
\end{tabular}

\section{Conclusão}

O sistema de fermentadores em série foi uma excelente ferramenta para avaliação de parâmetros fermentativos na elaboração de bebidas fermentadas, mantendo a completa anaerobiose e permitindo coletas assépticas e homogêneas segundo os padrões da literatura. 


\begin{abstract}
In pursuit of improving of apple's fermentation, the deployment of fermenters in series with different commercial strains of Saccharomyces cerevisiae: Arome Plus, Bouquet, Fermol Aromatic, manufacturer (AEB Group) and Saccharomyces cerevisiae UFLA CA - 11 in the preparation of fermented apples. Fermenters were made of glass with a volume of $1.45 \mathrm{~L}$ and $1.20 \mathrm{~L}$ working volume, with dimensions and materials that enable the autoclave sterilization. Fermenters were mounted in a room with controlled temperature $(20 \mathrm{C})$ and connected to a silicone's pipe saturation with carbon dioxide to maintain anaerobiosis with controlled pressure and flow of carbon dioxide, to allow collections through elevation of pressure in the system, an excellent tool for implementing the project. The system allowed us to evaluate each in quadruplicate under the same conditions for yeast fermentation The maximum sample collection represents $20 \%$ of total volume. This volume was sufficient for physical-chemical and microbiological tests during the fermentation period (10 days) until exhaustion of fermentable sugars. This fermentation's system was an excellent tool for assessing fermentative parameters in the preparation of fermented beverages.
\end{abstract}

Key-words: $\underline{\text { Saccharomyces }}$ sp., apples, fermentator, fermented.

\title{
Referências
}

ALBERTI, A.; NOGUEIRA, A.; WOSIACKI,G. Effect of the initial nitrogen content in apple wine processing. Brazilian Journal Food Technology. (in press), 2011.

BARON, A.; BOHUON, G.; DRILLEAU, J. F. Remarques sur l'indice formol des concentrés de jus de pomme. Annales des Falsifications, de l'Expertise Chimique et Toxicologique, v. 70, n. 749, p. 19-26, 1977.

BEECH, F. English cidermaking : technology, microbiology and biochemistry. Progress of Industrisl Microbiology, Amsterdam, v. 11, p. 133-213, 1972.

CSOMA, H.; SIPICZKI, M. Taxonomic reclassification of Candida stellata strains reveals frequent occurrence of Candida zemplinina in wine fermentation. FEMS Yeast Researche, v. 8, p. 328-336, 2008. DOI: 10.1111/j.15671364.2007.00339.x

DIERINGS, R. L. Abordagem microbiológica sobre as fermentações oxidativa, alcoólica e malolática no processamento da sidra. 114f. Dissertação (Mestrado em Ciência e Tecnologia de Alimentos) - Setor de Ciências Agrárias, Universidade Estadual de Ponta Grossa, Ponta Grossa, 2008.

DRILLEAU, J. F. Consolider les connaissances et maîtriser la qualité du produit fini. Pomme, n. 23, p. 23-25, 1991.

INSTITUTO ADOLFO LUTZ. Métodos físico-químicos para análise de alimentos. Coordenadores: ZENEBON, O.; PASCUET, N. S; TIGLEA, P. 1 ed digital. São Paulo:Instituto Adolfo Lutz, 2008. 1020 p.

NOGUEIRA, A.; MONGRUEL, C. N.; SIMÕES, D. R.; WOSIACKI, G. Effect of biomass reduction on the fermentation of cider. Brazilian Archives of Biology and Biotechnology, v. 51, n. 5, 2007.

NOGUEIRA, A.; LE QUERE, J. M.; GESTIN, P.; MICHEL, A.; WOSIACKI, G.; DRILLEAU, J. F. Slow fermentation in french cider processing due to partial biomass reduction. Journal Institute of Brewing, v.114, n. 2, p. 102-110, 2008.

PAGANINI, C.; NOGUEIRA, A.; DENARDI, F.; WOSIACKI, G. Análise da aptidão industrial de seis cultivares de maçãs considerando suas avaliações físico-químicas (Dados da safra 2001/2002). Ciência e Agrotecnologia, Lavras, v. 28, n. 6, p. 1336-1343, 2004.

PRETORIUS, I.S. Tailoring wine yeast for the new millennium: novel approaches to the ancient art of wine making. Yeast, v. 16, p. 675-729, 2000. DOI:10.1002/1097-0061(20000615)16:8<675::AID-YEA585>3.0.CO;2-B

SILVA, N.; JUNQUEIRA, V. C. A.; SILVEIRA, N.; TANIWAKI, M.; SANTOS, R. GOMES, R. Métodos de análise microbiológica de alimentos, 4. ed, São Paulo: Livraria Varella, 2010. 
VALERO, E.; SCHULLER, D.; CAMBON, B.; CASAL, M.; DEQUIN, S. Dissemination and survival of commercial wine yeast in the vineyard: A large-scale, three-years study. FEMS Yeast Research. v. 5, p. 959-969, 2005. DOI: 10.1016/j.femsyr.2005.04.007

WIECHETECK, F. V. B.; NOGUEIRA, A.; DRILLEAU, J. F.; WOSIACKI, G. Efeito da crioconcentração sobre o teor de compostos fenólicos em mostos industriais de maçãs. Publicatio UEPG. Ciências exatas e da terra, ciências agrárias e engenharias, Ponta Grossa - PR, v. 11, n. 1, p. 27-34, 2005.

WOSIACKI, G.; NAMIUCHI, N. N.; CERIBELLI, M. I. P. F.; SATAQUE, E. Y.; SICHIERI, V. L. F. S.; OLIVEIRA, T. C. R. M.; OLIVEIRA CESAR, E. Estabilidade do suco clarificado de maçã. Arquivos de Biologia e Tecnologia, Curitiba, v. 32, n. 4, p. 775-786, 1989.

Trabalho selecionado para apresentação oral durante a VIII SETAL- Semana de Tecnologia de Alimentos- Câmpus Ponta Grossa- Universidade Tecnológica Federal do Paraná- 01 a 03 de junho de 2011. Suplemento especial da RBTA. 